\title{
Flame Movements in Gaseous Explosions
}

P. ROF. W. A. BONE and Mr. R. P. Fraser have recently described their further experiments upon detonation of combustible gaseous mixtures.* Some very beautiful photographs of the phenomenon of 'spin', first observed by Campbell and Woodward (1926), have been taken by means of

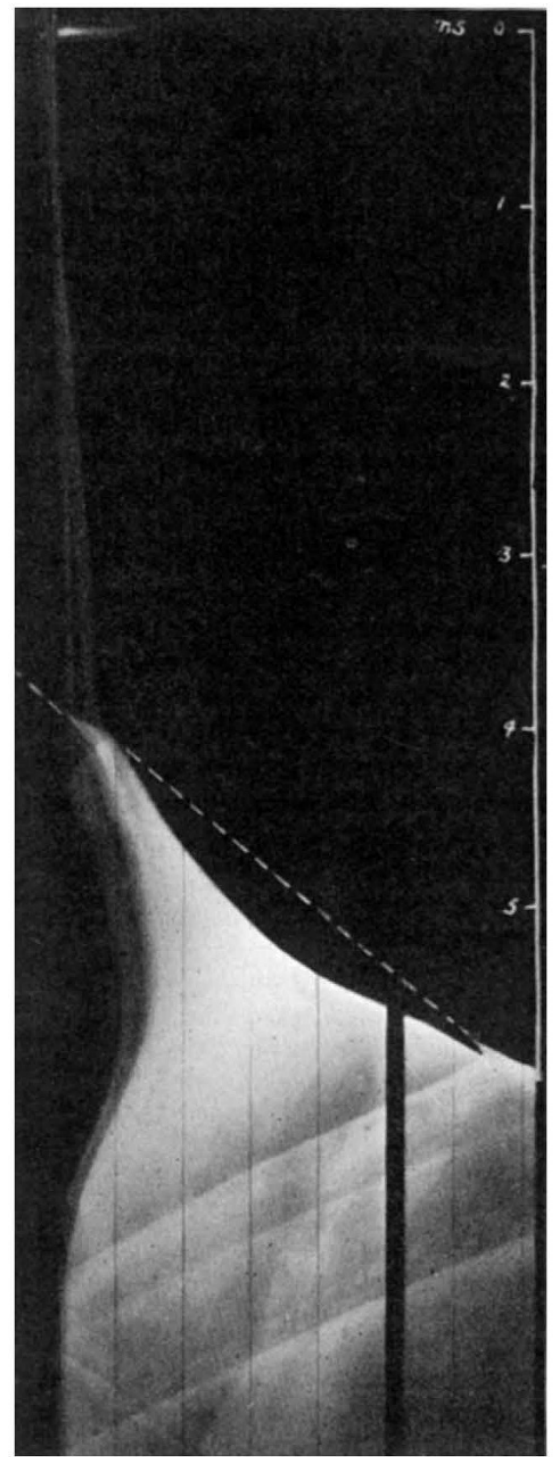

FIG. 1.-Compression wave (indicated by dotted line) overtaking advancing flame in $2 \mathrm{CO}: \mathrm{O}_{2}$ mixture.

the Fraser high speed camera, especially designed for this work, and described in Phil. Trans., vol. 228,1929, p. 197 . The larger machine was able to provide a vertical peripheral film speed up to 200 metres per second, so that resolution of the high speed movements of the flame front is obtainable.

Explosion of the gas mixtures in the long glass tubes was initiated either by a spark or by a high

* Photographic Investigation of Flame Movements in Gaseous Explosions, Parts iv., v., and vi., Phil. Trans., vol. 230, p. 363.

No. 3259 , VoL. 129] explosive detonator. Ignition could also be brought about farther down the tube, either by a spark or by means of a detonator which provided a shock wave in nitrogen contained in the first part of the tube. Depending on the strength of the detonator, the shock wave could either be allowed to follow the explosion flame, to catch it up, to proceed with the same speed, or to pass through it: it was shown that in the latter case the shock wave could initiate flame in the gas mixture ahead of the flame front, as is shown in Fig. 1. The flame is here travelling in the direction left to right, and the photographic paper moving rapidly in the upward direction at right angles to the direction of the flame. The sudden acceleration of the flame when the com.

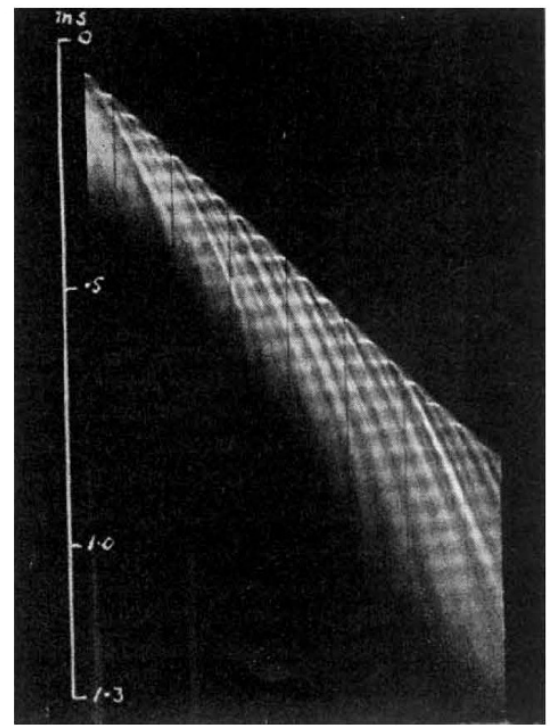

FIG. 2.-Spiral path of detonation in $2 \mathrm{CO}: \mathrm{O}_{2}$ mixture.

pression wave passes through it, and the subsequent ignition ahead of the compression wave prior to the arrival of the flame front, are clearly shown. The retonation wave can be seen travelling back through the burning gases, where the original flame front meets the auto-ignited flame. Impact of the shock wave on an advancing flame does not necessarily lead to detonation, but if the shock wave is sufficiently powerful, detonation is set up; it can even be caused to occur in this way close to the initiating spark. Prof. Bone and Mr. Fraser have confirmed that the velocity of the detonation wave during the early part of its course is more rapid than the subsequent uniform rate to which it settles down.

Part vi. of the paper deals with the phenomenon of spin already mentioned, which is well illustrated (Fig. 2). The flame does not appear to travel axially down the tube in the forward direction, but takes a spiral path: not only is this the case for the detonation wave, but also in some cases the phenomenon is observable in the advancing flame prior to detonation. Bands are visible in the gaseous 
products behind the flame, which have a frequency connected with the rotation of the "head" of detonation-frequencies of more than 100,000 a second being sometimes observed. The distance traversed per second by the head of detonation along its spiral path is constant in a given gas mixture, and the ratio of pitch to the diameter of tube is nearly constant, but in some cases two or more rotating ' heads ' of detonation are observable.

In the initial stages of an explosion the gaseous medium is moved forward along the direction of the tube axis : this forward impulsive motion would set up rotation about lines which may roughly be described as circles on a plane perpendicular to the axis and with centres approximately on it, and if this were superposed on an axial rotation a spiral vortex system would be initiated. Whether an explanation on this basis can be derived, or whether the rapid agitation of molecules through the gaseous medium sets up a disturbance along the axis, changing to a spiral wave front or 'head' which in turn gives rise to periodic waves in the 'tail' behind, are matters which are to be the subject of further investigations. Thirty-four photographs which Prof. Bone and Mr. Fraser have presented in their paper afford very considerable food for thought, and no such remarkable records of the behaviour of rapid flame motion have been pub. lished since those in Prof. H. B. Dixon's memoir in the Phil. Trans. of 1903.
A. C. E.

\section{Screen-Plate Colour Photography}

$\mathrm{T}$ HE two great lines of attack on the problem of colour photography are undoubtedly com prised in the "subtractive" processes on one hand and the "screen-plate" processes on the other. In spite of the initial simplicity of negative-making in the subtractive process and the application to three-colour work in commercial printing, the production of the following 'positive' colour pictures by the amateur is still a matter requiring some experience, skill, and patience. Although undoubted progress has been made, the results are not of a very certain character, and they are hard to control in any precise manner.

On the other hand, the 'screen-plate' processes are of a nature much more amenable to precise control. Their disadvantage, if it be a disadvantage, is that the immediate result is a transparency rather than a picture on paper; and the transparency cannot be seen to advantage without some care in its illumination or without the use of projection apparatus. We may, however, remark that the reason why so many amateurs nowadays are dissatisfied with their photographs is because the viewing of photographs has never received a tithe of the attention which has been so lavishly given to their taking. A small print made with a 4 -inch lens is viewed from a distance of twelve or fifteen inches. The result is a false rendering of the perspective and a general impression of an insignificant little picture. If the same photograph is printed as a transparency and viewed under proper illumination with a lens of 4-inch focal length, the perspective is seen under the proper angle and the result is vastly more satisfying and impressive. The projection of the picture is an equally good alternative.

The present writer has tried in vain to convince manufacturers upon this point, but if those who produce colour transparencies take the matter to heart they are not likely to tire of their pictures, even those made on a very small scale. Properly viewed or projected, transparencies have a wealth of beauty and satisfaction to give as compared with the finest pictures on paper.

The well-known Autochrome and Agfa plates have the colour mosaic incorporated in the first layer underneath the sensitive emulsion, and the positive picture is obtained by photographic 'reversal' of the negative obtained by exposure through the mosaic. The alternative procedure uses a colour screen printed separately on another plate of glass, the screen surface being held in contact with the emulsion of a panchromatic plate during the exposure. The negative resulting on development is printed as a positive and can then be bound up with a "viewing screen' containing a corresponding mosaic ; details are generally given in articles on colour photography. In this way, any number of copies can be made.

Examples of such processes were the Joly screen plate ruled in lines, and dating from 1904, and the plates developed by Mr. C. L. Finlay, which have been embodied in the 'Thames' and 'Paget' plates. The modern form is being marketed as the 'Finlay' process.

The progress made by photographic plate manufacturers has rendered possible corresponding progress in colour photography, since it is vitally dependent on satisfactory panchromatic emulsions. An Ilford panchromatic plate $(700 \mathrm{H}$ and $\mathrm{D})$ is specially coated for the Finlay process, and a series of five carefully adjusted compensating filters is available for use with different conditions of natural and artificiaI lighting. The high speed of the plates allows of snapshot exposures under favourable lighting conditions if a lens working at an aperture of $f / 4 \cdot 5$, or thereabouts, is used.

The "taking screen' with its mosaic of squares is printed by a secret process, but it is being claimed by the makers that a system of efficient inspection of the plates, and standardisation of the dyes, has enabled them to eliminate the variability which used to be experienced sometimes in the older types of plates. Methods of measurement of colour for microscopic objects are now easily available, and the efficient standardisation of the taking screens, filters, and viewing screens ought to be possible.

This is a matter of the very greatest importance for the technical use of the process in cases where pictorial colour records are required. The use of such records in microscopy, surgery, medical research, metallurgy, and other subjects would be

No. 3259 , VoL. 129] 